\title{
High rate production of nanopowders by the evaporation - condensation method using gyrotron radiation
}

\author{
A.V. Vodopyanov ${ }^{1}$,A.V. Samokhin ${ }^{2}$, N.V. Alexeev ${ }^{2}$, M.A. Sinayskiy ${ }^{2}$, A.I. Tsvetkov ${ }^{1}$, \\ D.A. Mansfeld ${ }^{1}$, M.Yu. Glyavin ${ }^{1}$, A.P. Fokin ${ }^{1}$ and V. I. Malygin ${ }^{1}$ \\ ${ }^{1}$ Institute of Applied Physics of Russian Academy of Sciences, Nizhny Novgorod, Russia, avod@yandex.ru \\ ${ }^{2}$ Baykov Institute of Metallurgy and Materials Science, Moscow, Russia
}

A new method for metal oxide nanopowder production was proposed recently by the authors $[1,2]$. It is the evaporation-condensation using a focused microwave radiation. The source of microwaves was the technological gyrotron with frequency of $24 \mathrm{GHz}$ and power up to $7 \mathrm{~kW}$ with the energy density flux of $13 \mathrm{~kW} / \mathrm{cm}^{2}$. Radiation was focused on a layer of powder of the treated material to ensure its evaporation, subsequent condensation of vapor in the gas stream, and deposition of particles on the water-cooled surface. A scheme of the experiment is shown in Fig. 1.

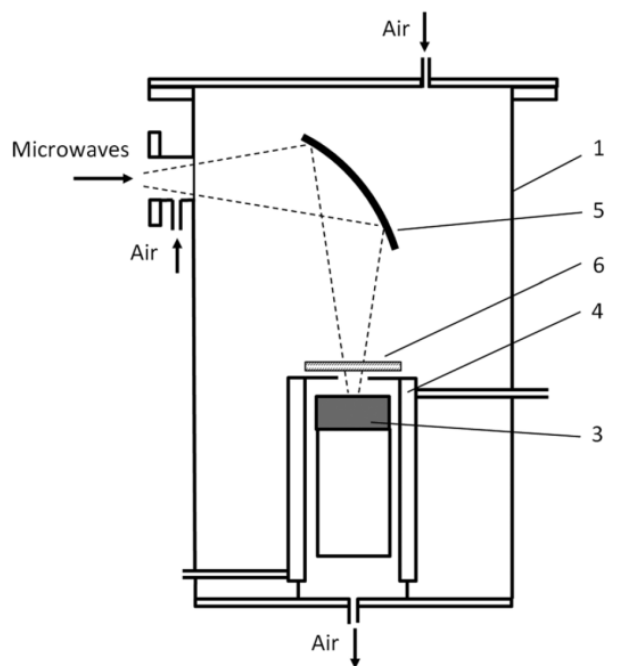

Fig. 1. Schematic view of the evaporation-condensation section: 1 - vessel, 2 - thermally insulating material, 3 - crucible with material, 4 - water-cooled collector, 5 - microwave focusing mirror, and 6 - quartz window

Deposited powders consist of particles whose sizes are in the range of $20 \mathrm{~nm}$ to $1 \mu \mathrm{m}$. The powder consists of particles which have different shapes - close to spherical shape as well as octahedral, which indicates that the mechanism of particles formation is "vapor-liquid-crystal" as well as "vapor-crystal." Fig. 2 shows examples of nanopowders obtained. The produced powders are the ensembles of particles whose sizes are in the range of $20 \mathrm{~nm}$ to $1 \mathrm{um}$. The $\mathrm{WO}_{3}$ powders consist of particles having different shapes close to spherical shape as well as octahedral, which indicates that the mechanism of particles formation is vapor-liquid-crystal as well as vaporcrystal. The formation of the nanoparticles in evaporation-condensation processes occurs by vapor condensation and it is determined by temperature conditions, which in turn depends on the flux density of microwave energy and velocity distribution of the gas incident on the evaporation surface. If the particle growth is from the vapor phase at temperatures above the melting point, the particles are spherical. The deposited tin oxide powder consists of particles with sizes of 20-200 nm, preferably with equiaxed shape and a facet. The obtained zinc oxide powder has a complex morphology consisting mainly of two types of particles-faceted rods with the length of 200-300 nm and a transverse dimension of about $60 \mathrm{~nm}$ and nanowhiskers of approximately the same length but with a transverse dimension not greater than $20 \mathrm{~nm}$. The maximum evaporation rate was $100 \mathrm{~g} / \mathrm{hr}$. Evaporation rate depending to microwave power is shown in Fig. 3.

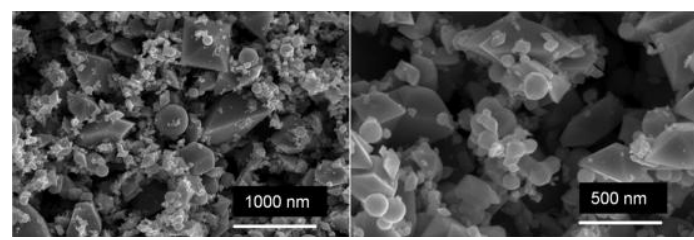

(a)

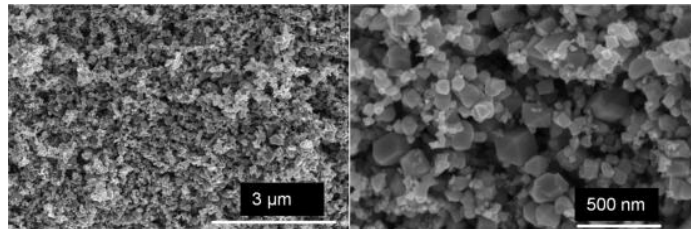

(b)

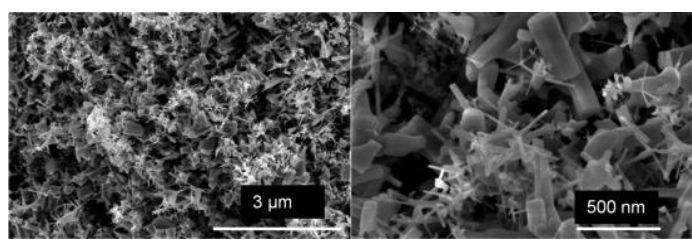

(c)

Fig. 2. Microscope images of powders.

(a) $-\mathrm{WO}_{3}$, (b) $-\mathrm{SnO}_{2}$, (c) $-\mathrm{ZnO}$

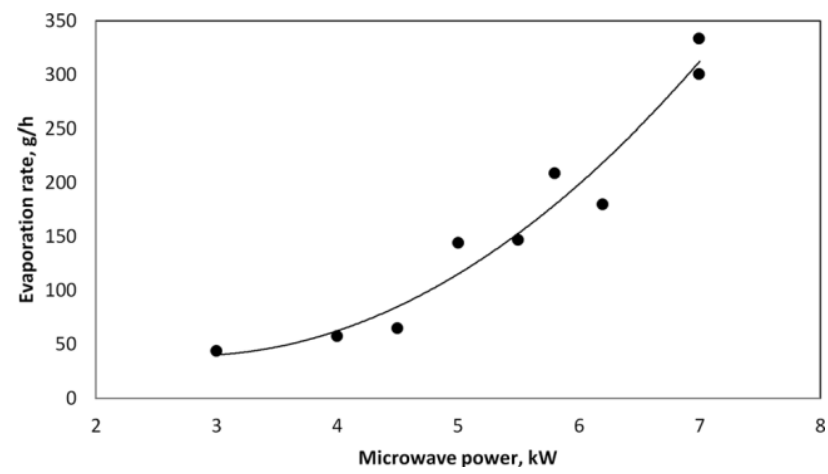

Fig. 3. Evaporation rate of $\mathrm{WO}_{3}$ versus microwave power

This method was upgraded by the use of a subterahertz gyrotron setup with output frequency of $263 \mathrm{GHz}$ and a nominal power of $1 \mathrm{~kW}$ as a radiation source. In 
this case microwave-energy flux density was of about $20 \mathrm{~kW} / \mathrm{cm}^{2}$. A significant increase in the substance evaporation rate compared with the previous experiments has been shown. But in this case evaporation rate is slightly decreasing with time, see Fig. 4.

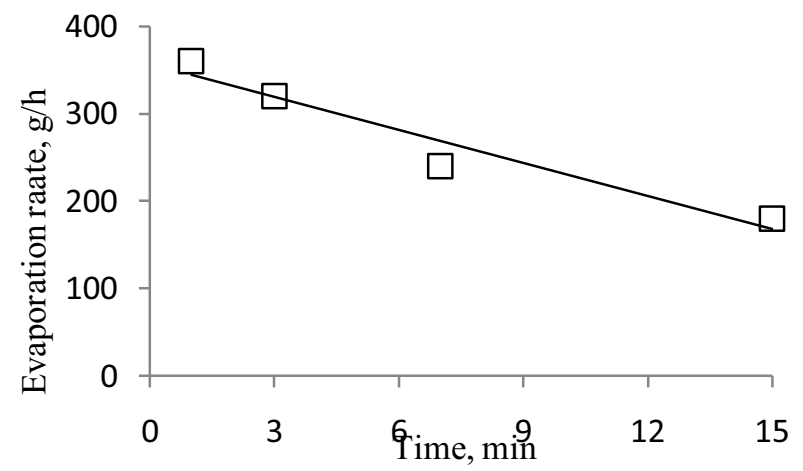

Fig. 4. Time dependence of the evaporation rate for $\mathrm{WO}_{3}$

This is due to the formation of the increasing crater at the place of action of the microwave beam on the sample. On the one hand, this leads to a change in the flow pattern of the quenching gas in this region and, on the other hand, reduces the power density of the microwave beam on the heated surface due to its leaving of the focal waist. These drawbacks can be partly compensated by using a mechanical system of height adjustment of a crucible with the sample and upgrading the air flow system.

The experimental results demonstrated that the size of nanoparticles could be adjusted by tuning the heating power - cooling air flow ratio. Fig. 5 shows the typical dependence of the specific surface of the obtained powder on the quenching air flow rate.

A significant increase in the substance evaporation rate compared with similar experiments using a technological gyrotron setup with a frequency of $24 \mathrm{GHz}$ as a radiation source was demonstrated. An increase in the radiation frequency leads to the improvement of the heating efficiency due to an increase of the absorption coefficient and provides a greater power density due to a better focusing of the radiation. The sizes of the nanoparticles obtained in the experiments range from 20 to $500 \mathrm{~nm}$. The maximum rate of evaporation of tungsten trioxide $\mathrm{WO}_{3}$ was about $360 \mathrm{~g} / \mathrm{h}$ and of zinc oxide $\mathrm{ZnO}$, about $50 \mathrm{~g} / \mathrm{h}$.

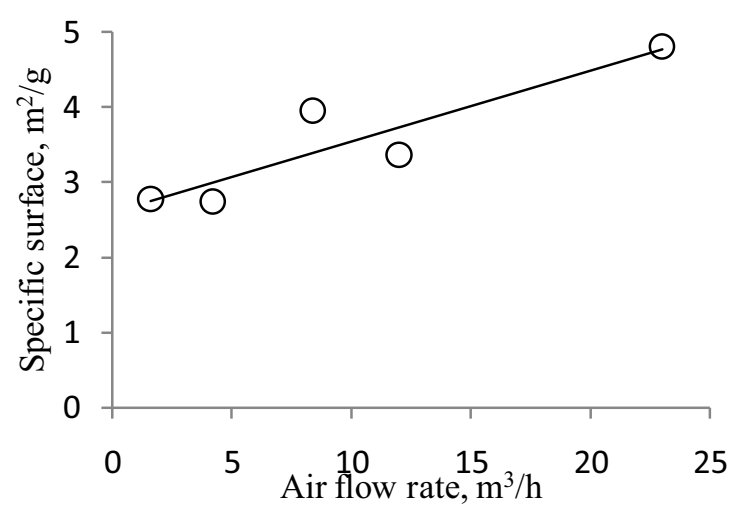

Fig. 5. Specific surface of the obtained powder on the air flow rate for tungsten trioxide $\mathrm{WO}_{3}$

\section{Acknowledgements}

The work was supported by the Russian Science Foundation, project No. 15-12-00046.

\section{References}

1. A. V. Samokhin et al., "Production of WO3 tungsten oxide nanopowders by evaporation-condensation process using focused 24-GHz microwave radiation," High Energy Chem., vol. 49, no. 4, pp. 267-272, 2015.

2. A. V. Samokhin, N. V. Alexeev, A. V. Vodopyanov, D. A. Mansfeld, and $Y$. V. Tsvetkov, "Metal Oxide Nanopowder Production by Evaporation- Condensation Using a Focused Microwave Radiation at a Frequency of $24 \mathrm{GHz}$," J. Nanotechnol. Eng. Med., vol. 6, no. 1, p. 11008, 2015. 